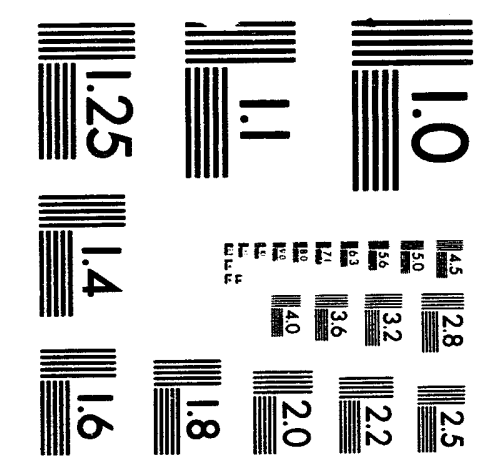



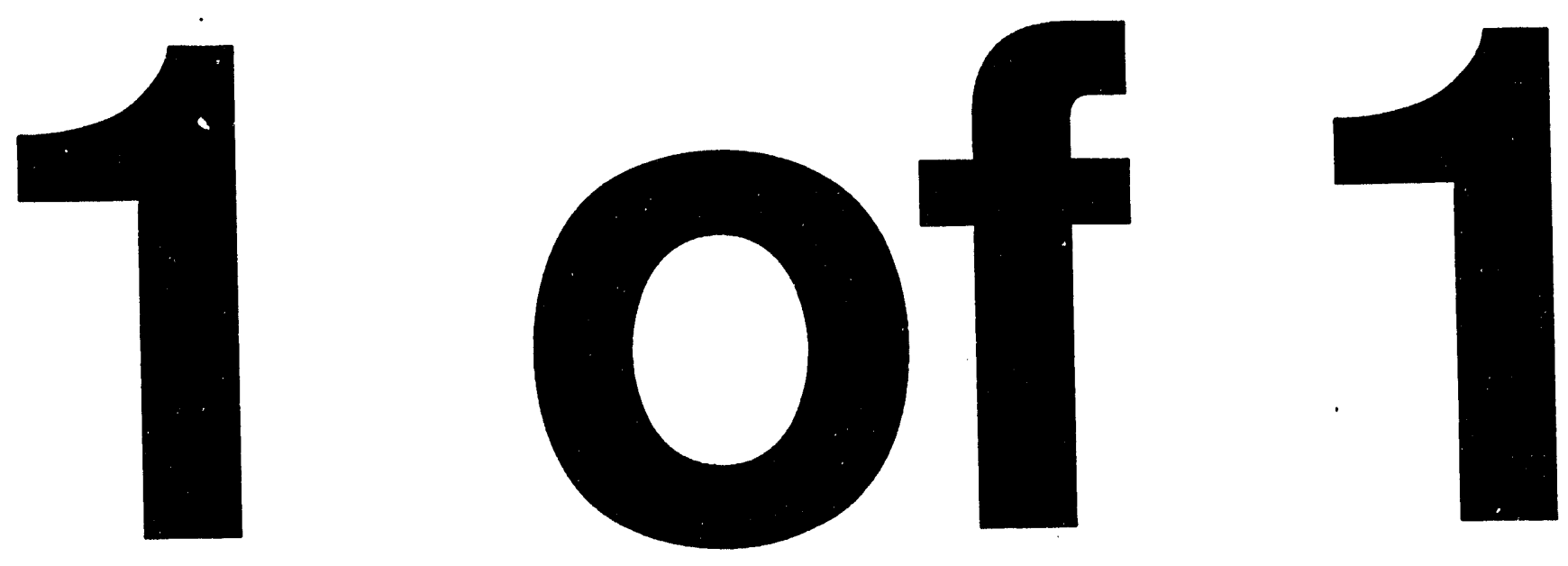
LBL-33998

UC-414

Presented at the IEEE 1993 Nuclear Science Symposium and

Medical Imaging Conference, San Francisco, California, October 31 - November 6, 1993.

To be published in the IEEE Trans. on Nucl. Sci., June 1994

\section{MICROSTRIP GAS CHAMBERS ON GLASS AND CERAMIC SUBSTRATES}

W.G. Gong, H.Wieman, J.W. Harris, W.S. Hong, J.T. Mitchell, V. Perez-Mendez Lawrence Berkeley Laboratory University of California, Berkeley, CA 94720

November 1993

This work was supported by the Director, Office of Energy Research, Division of Nuclear Physics of the Office of High Energy and Nuclear Physics of the U.S. Department of Energy under Contract DE-AC03-76SF00098 


\title{
MICROSTRIP GAS CHAMBERS ON GLASS AND CERAMIC SUBSTRATES*
}

\author{
W.G. Gong, H. Wieman, J.W. Harris, J.T. Mitchell, \\ Nuclear Science Division, and \\ W.S.Hong, V. Perez-Mendez, \\ Physics Division, \\ Lawrence Berkeley Laboratory, Berkeley, CA94720, USA
}

\section{Abstract}

We report developments of Microstrip Gas Chambers (MSGC) fabricated on glass and ceramic substrates with various resistivities. Low resistivity of the substrate is found to be critical for achieving stable operation of microstrip gas chambers. The microstrip pattern consists of $10 \mu \mathrm{m}$ wide anodes and $90 \mu \mathrm{m}$ wide cathodes with a 200 1 $\mathrm{m}$ m anode-to-anode pitch. High-quality microstrips are fabricated using the dry etch after UV-photolithography. Our chambers are tested in an $\mathrm{Ar}(90)-\mathrm{CH}_{4}(10)$ gas mixture at atmospheric pressure with a $100 \mu \mathrm{Ci}{ }^{55} \mathrm{Fe}$ source. An energy resolution (FWHM) of $15 \%$ has been achieved for $6 \mathrm{keV}$ soft $X$-rays. At a rate of $5 \times 10^{4}$ photons $/ \mathrm{sec} / \mathrm{m}^{2} \mathrm{~m}^{2}$, gas gains are stable within a few percents. Long-term tests of gain stability and rate capability are yet to be pursued.

\section{INTRODUCTION}

The introduction of the Microstrip Gas Chamber (MSGC) by $A$. Oed [1] has attracted a great deal of attention over the last five years. Many efforts [2-13] have demonstrated that this new gas detector has much improved performance over a conventional Multi-Wire Proportional Chamber (MWPC). Its energy resolution, spatial resolution, rate capability radiation resistance, and low cost make it suitable for a wide range of applications, e.g. neutron diffraction in material analysis [3], X-ray imaging in both astronomy [6] and synchrotron radiation [8], chargedparticle tracking in high-energy particle physics and nuclear physics $[7,12,13]$.

The Microstrip Gas Chamber is a miniaturized type of the Multi-Wire Proportional Chamber with the wires being replaced by thin-film strips and one cathode drift plane being replaced by an insulating substrate to support the strips. A typical structure of the MSGC is shown in Fig. 1. It consists of a drift region and a gain region. The drift region is defined by the gas volume between the drift cathode plane and the microstrip plate. The gain region is defined

-This work was supported by the U.S. Department of Energy, under contracts No. DE-A.C03-76SF00098.

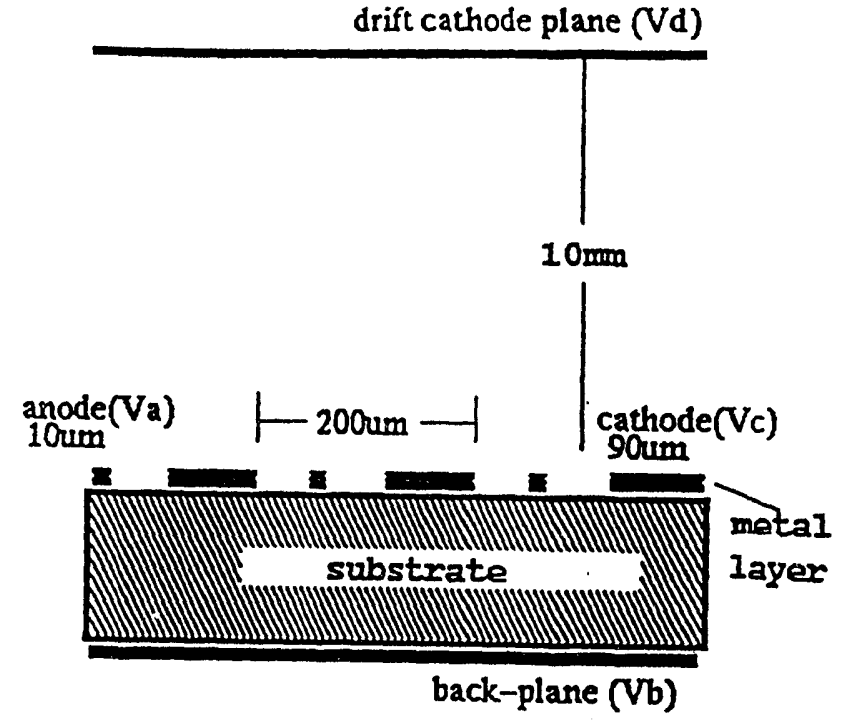

Figure 1: The structure of a MSGC.

by an array of $10 \mu \mathrm{m}$-wide anode strips and $90 \mu \mathrm{m}$-wide cathode strips at a pitch of $200 \mu \mathrm{m}$. The microstrips are supported by an insulating substrate. Electronic signals induced by ionizing radiation traversing the gas volume can be read out from either anodes or cathodes, or even the back-plane if the substrate is sufficiently thin. An $11 \%$ energy resolution (FWHM) for $6 \mathrm{keV}$ soft X-rays (using $3 \mu \mathrm{m}$ wide anodes), a $30 \mu \mathrm{m}$ spatial resolution for minimum ionizing particles, a $10^{6}$ particles $/ \mathrm{sec} / \mathrm{mm}^{2}$ rate capability, and a 9 ns time resolution (rms) has been achieved [7].

The performance of the MSGC can be hindered by timeand/or rate-dependent instabilities of gas gain due to surface charging on the insulator. It was realized that the surface resistivity of the substrate must be low enough to quickly neutralize the positive avalanche ions accumulated on the insulator surface. On the other hand, the surface resistivity should be high enough to maintain the high bias voltage and low leakage current across the anodes and cathodes. Though expensive, ion implantation of high dosage $\left(\sim 4 \times 10^{16}\right.$ ions $\left./ \mathrm{cm}^{2}\right)$ was effective in lowering the surface resistivity of quartz or $\mathrm{SiO}_{2}$ film in order to keep the gain of the MSGC stable at high rate[7]. Another approach is to use directly a glass substrate of low 
resistivity and electronic conductivity $[3,10,11]$.

In recognizing the possibility to use the MSGCs as readout for a Time Projection Chamber (TPC) for trarling charged particles, we began to study the performance of the MSGC for this purpose at the Lawrence Berkeley Laboratory. In this study, we have learned how to build the Microstrip Gas Chambers and how to operate them. We have investigated the correlation between substrate resistivity and gain stability. Two types of low resistive glass are used: Schott 88900 and Corning 7740. One type of ceramic substrate (Alumina) is also used with B-ion implantation. We present characterizations of microstrip plates, pulse height spectra for $6 \mathrm{keV}$ soft X-rays, gas gain dynamic ranges, and time-dependent gain stability for the three substrates.

\section{FABRICATION OF MSGC}

One distinct feature of the Microstrip Gas Chamber is that it is fabricated using microelectronics technology. These techniques can produce highly uniform strips with sub-micron precision, which eliminate wire instability and placement inaccuracy in a multiwire chamber with submillimeter pitch. By using the microfabrication technique, one can build a gas chamber with a great improvement in detector performance.

We have used the Berkeley Microfabrication Laboratory to fabricate our microstrip plates. It is a self-service laboratory operated by the Department of Electrical Engineering and Computer Science in the University of California at Berkeley. This microlab is equipped with standard facilities for microelectronics processing such as wafer cleaning sink, mask generator, high-temperature furnaces for Chemical Vapor Deposition (CVD) and thermal processing, thin-film metallic coating system, photolithography station, plasma etcher, analytical and packaging tools, and computers.

Fig. 2 shows the steps we followed in fabricating MSGCs. Step (a): The substrate is cleaned in a wetsink so that the resistivity monitor reads above $12 \mathrm{M} \Omega \cdot \mathrm{cm}$;

Step (b): A $2 \mu \mathrm{m}$ layer of $\mathrm{Al}$ or $\mathrm{Au}$ thin film is coated on the substrate by means of sputtering or evaporation;

Step (c): A $1.5 \mu \mathrm{m}$ layer of photoresist (PR) is spin-coated on the metallic film followed by a brief softbake at $90^{\circ}$;

Step (d): A microstrip mask is aligned on a lithography station under microscope and the photoresist layer is then exposed to the ultra-violet light through the mask;

Step (e): The microstrip pattern is transferred onto the metallic layer after developing the photoresist film. The unexposed photoresist is then hardened by a 30-minuite hardbake at $120^{\circ}$;

Step (f): The exposed metallic film is removed with a dry etch;

Step $(\mathrm{g})$ : The photoresist film is stripped off by either Acetone or $\mathrm{O}_{2}$ plasma to obtain the final microstrip plate.

We note that (1) the substrate can be glass, ceramics, (a) Glass

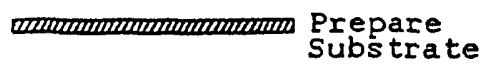

(b) Hetal

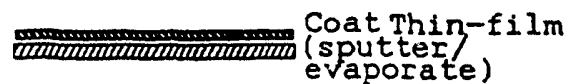

(c) Resist Metal

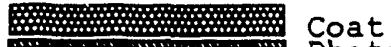
Glass

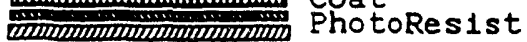

(d)
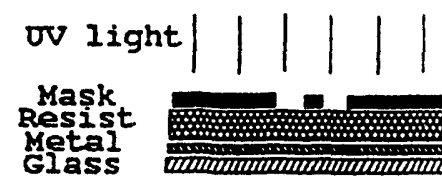

Align Mask \& Expose to UV

(e)

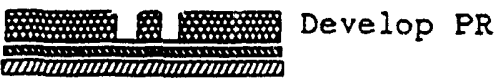

(E)

Chlorine Argon

Argon
Ion-beam $|1|$ (Plasmal

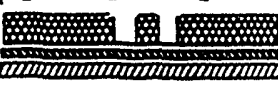

(g) MSGC

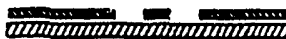

Ash/Strip PR

Figure 2: Steps for fabricating a MSGC.

or $\mathrm{Si} / \mathrm{Quartz}$ wafers as long as they have smooth surfaces; (2) Wet etch can also be used in Step (f). However, it is known that dry etch is superior to wet etch. Wet etch is isotropic and can produce undercut and non-uniform strips. In contrast, dry etch is anisotropic and can yield strips of better uniformity and edge profile. We have used two types of dry etch: reactive plasma and inertia ionbeam. In the first case, Chlorine plasma is used to etch an Al film through chemical reactions, which is selective to the metallic material. In the second case, an Argon ionbeam is used to etch Au film by surface abrasion, a process that has no selectivity and works with any material.

Fig. 3 shows two photographs of microstrips obtained by dry etch at two magnification scales. The width of thin anode strips is $10 \mu \mathrm{m}$. Fig. 4 gives a cross-section view of the microstrips at two different horizontal scales taken by a stylus profiling device. It is obvious that the dry etch produces strips of high quality in terms of uniformity and edge profile. We have therefore used dry etch for our work.

\section{EXPERIMENTAL SetuP}

Our microstrip mask consists of 40 strips of $1.9 \mathrm{~cm}$ in length and $0.8 \mathrm{~cm}$ in width. Each anode is $10 \mu \mathrm{m}$ wide and each cathode is $90 \mu \mathrm{m}$ wide. The anode-to-anode pitch is $200 \mu \mathrm{m}$ as depicted in Fig. 1. The drift plane is made of a thin Aluminized-Mylar foil. It is located 10 $\mathrm{mm}$ away from the microstrip plate. The plate is mounted on a printed circuit board inside an Aluminum chamber 


\begin{tabular}{|c|c|c|c|}
\hline Name/Supplier & Composition(\%) & Resistivity & Comment \\
\hline S8900/Schott Glass & $\begin{array}{c}\text { Silica/Barium Oxide(20-50), } \\
\text { Iron Oxide(10-20), Vanadium/ } \\
\text { Sodium/Strontium Oxide(1-10) }\end{array}$ & $\begin{array}{c}\rho_{v}=1.4 \times 10^{11} \Omega \mathrm{cm} @ 22^{\circ} \mathrm{C} \\
\rho_{s}=3 \times 10^{13} \Omega / \square\end{array}$ & $\begin{array}{c}\text { smooth surface } \\
\text { special order }\end{array}$ \\
\hline C7740/Corning Glass & $\begin{array}{c}\text { Silica(80), Boric Oxide(14) } \\
\text { Soda(4), Alumina(2) }\end{array}$ & $\begin{array}{c}\rho_{v}=10^{15} \Omega \mathrm{cm} @ 25^{\circ} \mathrm{C} \\
\rho_{s}=3 \times 10^{15} \Omega / \square\end{array}$ & $\begin{array}{c}\text { smooth surface } \\
\text { regular order }\end{array}$ \\
\hline A445/Kyocera Ceramics & Alumina(91) & $\rho_{v}=10^{12} \Omega \mathrm{cm} @ 20^{\circ} \mathrm{C}$ & $\begin{array}{c}\text { porous surface } \\
\text { (unusable) }\end{array}$ \\
\hline A201/Kyocera Ceramics & Alumina(98) & $\begin{array}{c}\rho_{s}=1.6 \times 10^{16} \Omega / \square \\
10^{16} \text { ions } / \mathrm{cm}^{2}(150 \mathrm{keV} \text { B) }\end{array}$ & $\begin{array}{c}\text { smooth surface } \\
\text { optically polished }\end{array}$ \\
\hline
\end{tabular}

Table 1: Properties of glass and ceramic substrates used in this work.
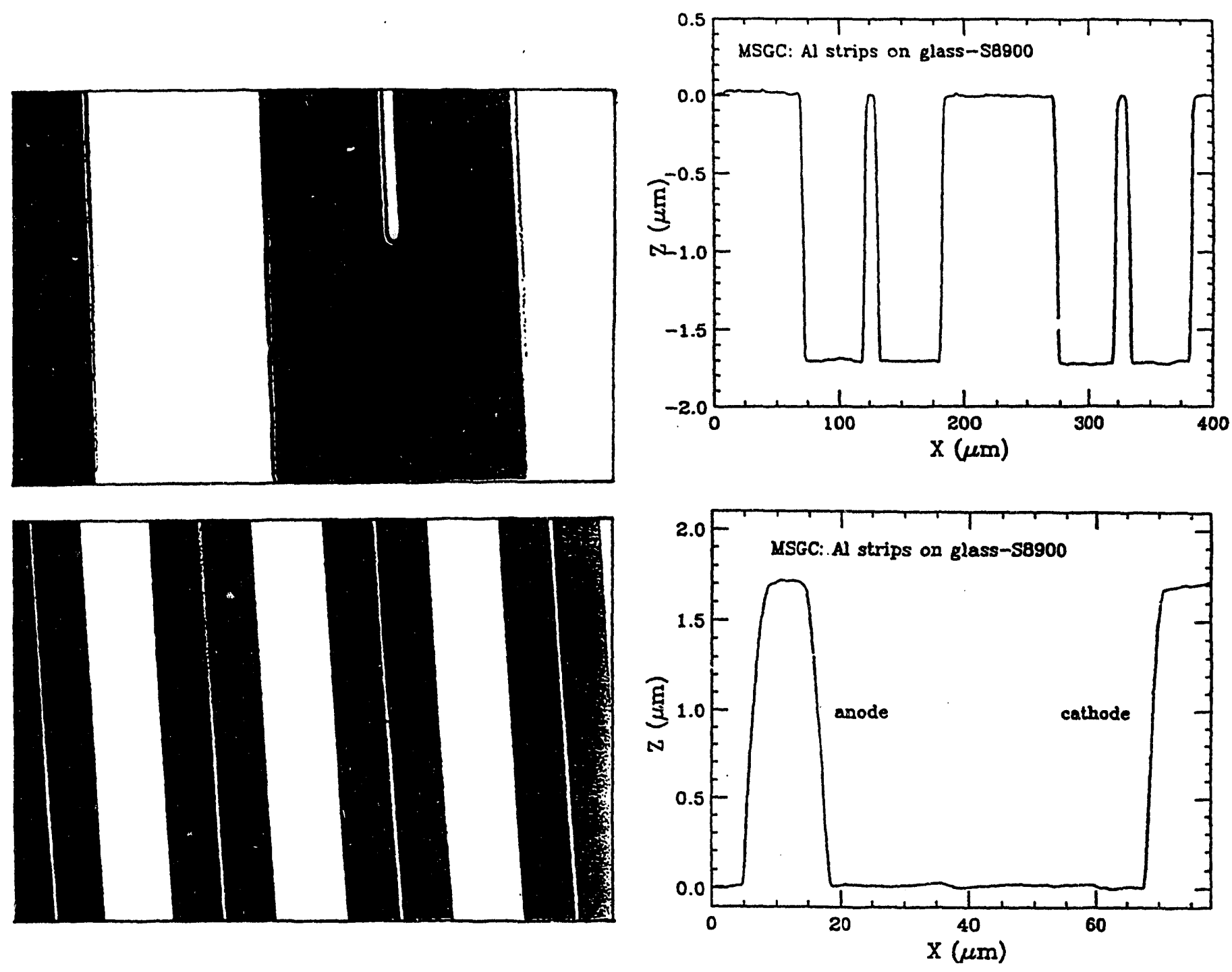

Figure 3: Photographs of microstrips obtained by the dry etch. The upper picture has larger magnification. The width of thin strips is $10 \mu \mathrm{m}$.

Figure 4: Cross-section views of microstrips obtained by the dry etch. 


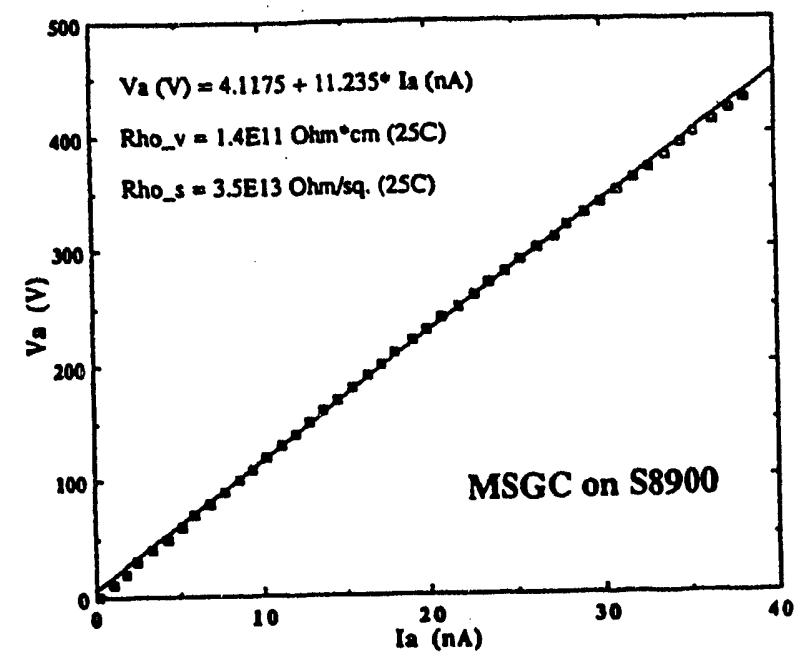

Figure 5: The leakage current measured with 4 anode strips on Schott glass S8900.

with an O-ring seal. P-10 gas (90\% Argon (Ar) and 10\% Methane $\left(\mathrm{CH}_{4}\right)$ ) is used. The chamber humidity is monitored using a hygrometer. Before the test, we inspect all anode and cathode strips with a microscope. Four contiguous anode strips are connected together and are biased at a positive high-voltage through a current-limiting resistor. All cathode strips are interconnected and held at ground potential. To reduce discharge and its damage to strips, epoxy or photo-definable polyimide thin-films are applied to the end region of strips. The anode readout is capacitively coupled to a charge-sensitive preamplifier which is followed by a shaping amplifier. The pulse height is digitized by a peak-sensing ADC in a CAMAC crate. The data acquisition system is the Kmax-3.1 installed on a Macintosh computer. A $100 \mu \mathrm{Ci}^{55} \mathrm{Fe} \mathrm{X}$-ray source is used and placed on a hole collimator of $1 \mathrm{~mm}$ in diameter above the anode strips. The X-ray spectra are recorded to study energy resolution, gain dynamic range, and time dependent stability for different microstrip plates.

\section{MSGC on Glass Substrate}

Glass substrates have been widely used for making MSGCs $[1,3-6,8-13]$. Glass has a very smooth surface and is suitable for photolithography processing. Recent work has shown that electronic conduction in the glass is responsible for achieving stable operation and high rate capability for a MSGC[9-11]. We have used two types of glass: Schott S8900 and Corning 7740 (see Table 1). S8900 is a special glass containing a high compositions of Barium and Iron oxide, and a small amount of Vanadium/Strontium oxide. It has low resistivity and has been used in the Pestov Spark Chambers. As shown in Fig. 5, the leakage current is linear with the bias voltage. This ohmic behavior is indicative of electronic conduction in the glass. Soda Lime glass also has unusually low resistivity $\left(\log \left(\rho_{v}[\Omega \mathrm{cm}]\right)=12.4 @ 25^{\circ} \mathrm{C}\right)$.
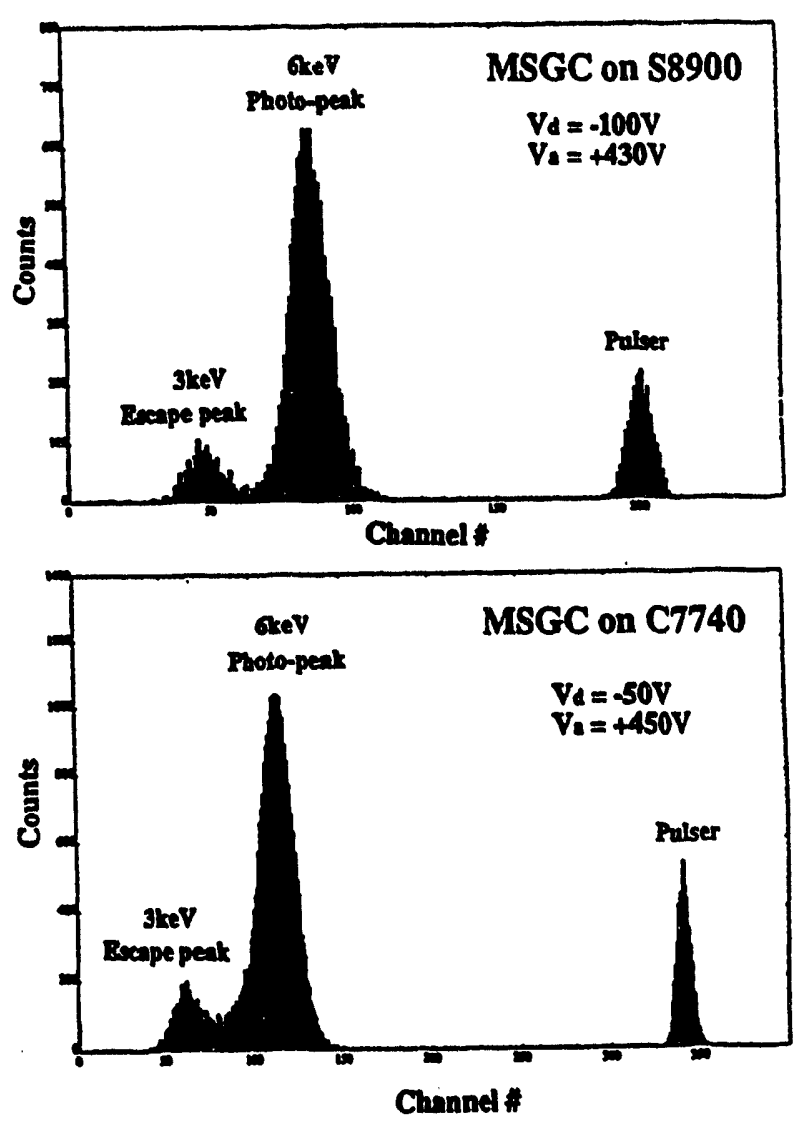

Figure 6: Pulse height spectra of ${ }^{55} \mathrm{Fe} \mathrm{X}$-rays measured by the MSGC on 88900 (upper) and by the MSGC on C7740 (lower), respectively, in P-10 gas. Bias voltages are indicated in the figures.

However, it is ionic-conductive due to its high amount of Sodium ions. Its resistivity is unstable under high-voltage bias and is not suitable for the MSGC. C7740 is a Borosilicate glass. Although it has somewhat higher resistivity, it is stable and is also readily available. We fabricated microstrips on both glasses. On the $\$ 8900$ glass, an Al thin-film of $1.7 \mu \mathrm{m}$ thickness is coated by sputtering and etched by Chlorine plasma. On the C7740 glass, a Au thinfilm of about $2 \mu \mathrm{m}$ is coated by thermal evaporation and etched by ion-milling with Ar ion-beams. Figs. 3 and 4 show the actual quality of microstrips on $\mathbf{S} 8900$ glass. The quality of microstrips on $\mathrm{C7740}$ glass is similar (not shown here).

As shown in Fig. 6, we have obtained pulse height spectra for soft X-rays from a collimated ${ }^{55} \mathrm{Fe}$ source $(100 \mu \mathrm{Ci})$. The upper half of the figure shows the spectrum of $X$-rays measured using the MSGC fabricated on the $\$ 8900$ glass. The drift cathode bias is $-100 \mathrm{~V}$ and the anode bias is +430 $V$ while the cathode strips are grounded. Both the photopeak at $6 \mathrm{keV}$ and the escape peak at $3 \mathrm{keV}$ are clearly resolved and well separated. A pulser peak is also shown to indicate the electronic noise level. The FWHM of the pulser peak is 10 channels, and the FWHM of the photopeak is 15 channels. After removing the electronic noise contribution to the peak width, the resolution (FWHM) 


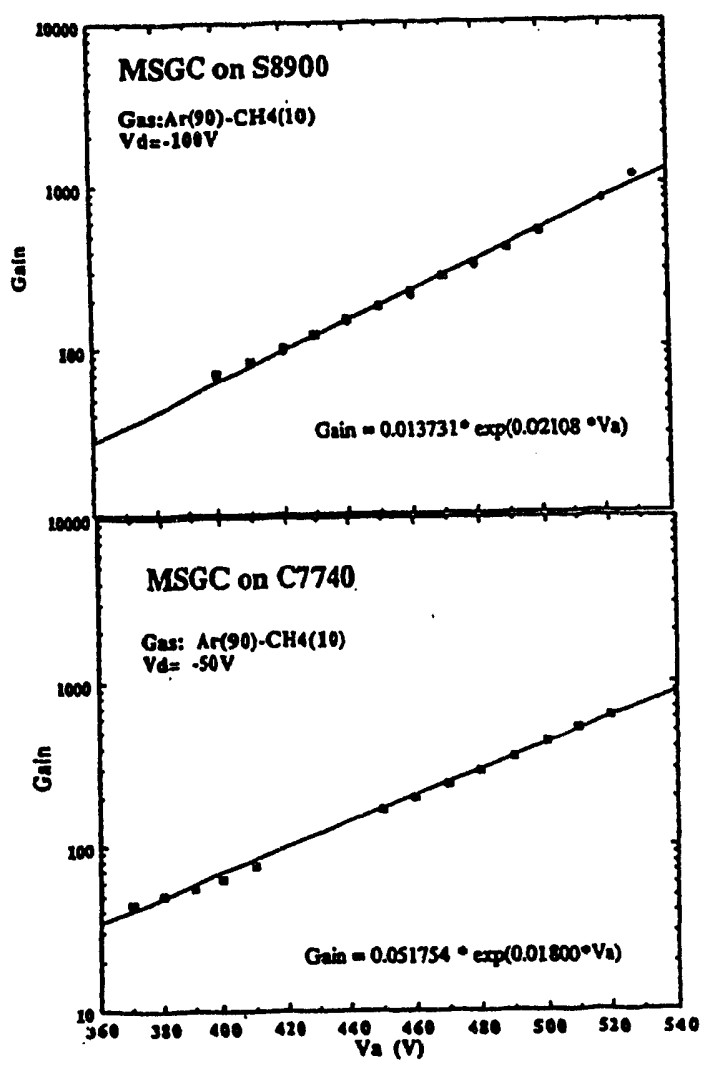

Figure 7: The gas gain dependence on anode voltages for the MSGC on S8900 (upper) and for the MSGC on C7740 (lower), respectively, in P-10 g\%. Exponential fits are shown.

for the $6 \mathrm{keV}$ peak is $14.7 \%$. The lower panel shows the $\mathrm{X}$-ray spectrum measured by the MSGC on C7740. The drift cathode bias is $-50 \mathrm{~V}$ and the anode bias is $+450 \mathrm{~V}$ with the cathode grounded. Both the photo-peak and the escape peak are also resolved even though the FWHM resolution seems to be slightly worse $(\mathbf{1 8 . 8 \% )}$. This could be related to the differences in etching process or test condition. In general, both dry-etch processes produce highly uniform strips and should give rise to good energy resolutions.

We have measured the photo-peak channel numbers for anode voltages ranging from $+360 \mathrm{~V}$ to $+530 \mathrm{~V}$ to study the dynamic range of gas gains. The channel numbers are then converted to collected charges in coulombs, which is calibrated by injecting a given amount of charge at the preamplifier input through a $0.5 \mathrm{pF}$ test capacitor. (We comment that a larger capacitor should have been used in order to reduce stray-capacitor contribution. The gas gains may be under-estimated here.) It is known that the average ionization potential of P-10 gas is $26 \mathrm{eV}$. The gas gain is defined by the ratio of collected charges over the primary charges. Fig. 7 shows the gas gains measured for the MSGC on S8900 (upper) and for the MSGC on C7740 (lower), respectively. Exponential fits can represent the data points very well indicating a good proportionality for the microstrip gas chamber. Both chambers have
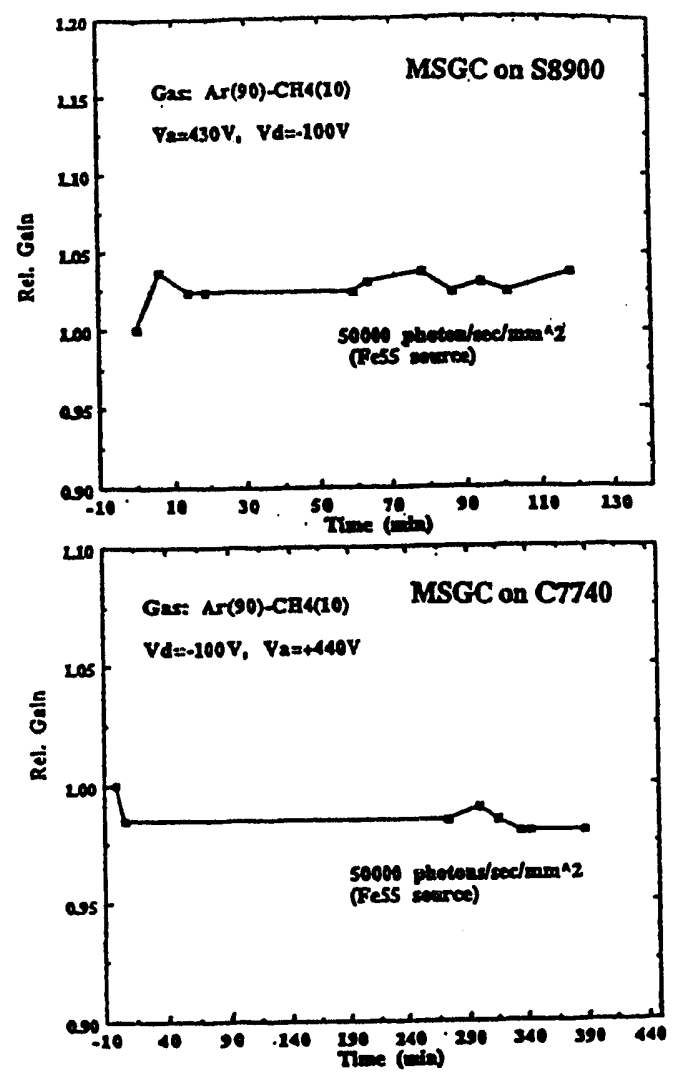

Figure 8: Time dependence of the relative gas gains for the MSGC on S8900 (upper), and for the MSGC on C7740 (lower), respectively. They are monitored by a ${ }^{55} \mathrm{Fe}$ source with a flux of 50000 photons $/ \mathrm{sec} / \mathrm{mm}^{2}$. All bias voltages are indicated.

similar gas gain ranging from 30 to 1000 . The maximum gas gains are limited by discharge breakdown at anode voltages around $530 \mathrm{~V}$. Recent work $[14,15]$ suggests that much higher gain can be obtained either by using more quenching \& \& such as DME or Ethane, or by passivating the MSGC with a very thin layer of Nickel oxide coating.

The surface-charging effect has been an important issue in developing a microstrip gas chamber from the very beginning. Because the strong electric fields are concentrated on the insulator surface, most of positive ions formed during avalanches will drift from anode strips to nearby cathode strips (typically $50 \mu \mathrm{m}$ away) while very few ions will go to the drift cathode plane (typically several $\mathrm{mm}$ away). The reduction of spaçe charges leads to a higher rate capability of a microstrip gas chamber than that of a multiwire proportional chamber. However, surface charge can affect the MSGC's performance significantly. When positive ions are accumulated on the insulator surface, they distort the electrical fields around strips. If they cannot be neutralized before the next avalanche event, the gas gain will be reduced thereafter. Both rate dependence and time dependence of gas gain can be related to the same surfacecharge effect. For the time being, we only measured the time dependence on a relatively short term using a radioactive source. We plan to study time dependence on longer 


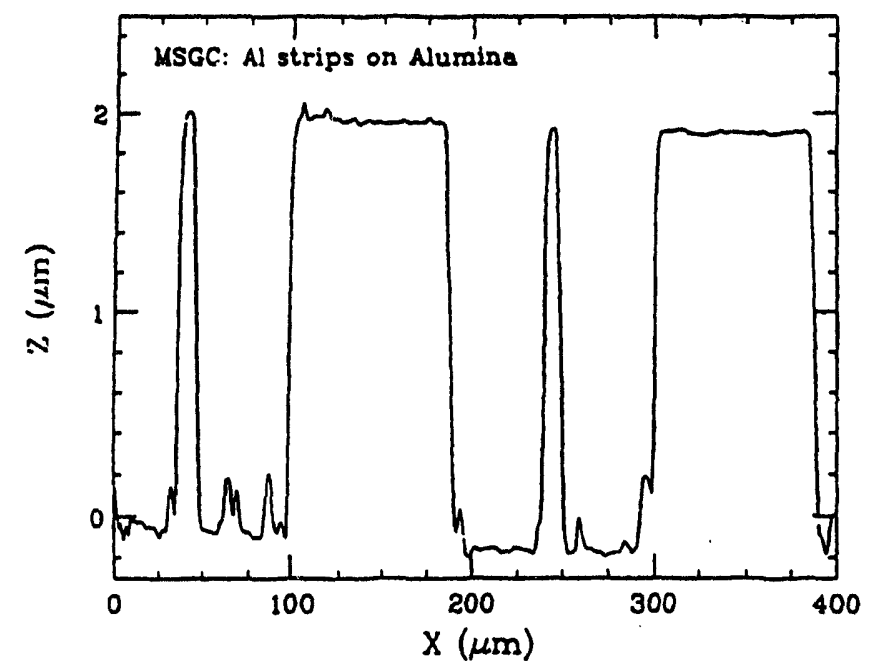

Figure 9: A cross-section view of anode and cathode strips fabricated on the ceramic substrate.

term and rate dependence with an X-ray machine later. Fig. 8 displays the relative gain stability for the MSGC on S8900 (upper) within 2 hours, and for the MSGC on C7740 (lower) within 7 hours, respectively, using an ${ }^{55} \mathrm{Fe}$ source of flux equal to 50000 photons $/ \mathrm{sec} / \mathrm{mm}^{2}$. After the first 10 minuites of conditioning, gas gains are stable within $2 \%$ for both chambers. Even though the rate of $\mathrm{X}$-rays are moderate, the performance of our MSGCs are consistent with the more systematic work done by Sauli's group at CERN[10].

\section{MSGC on Ceramic Substrate}

Extensive work[3, 9-13] suggests that a microstrip gas chamber of good performance can be built on a glass substrate of electronic conductivity. One question concerning detecior packaging needs more investigations. How to bridge charge signals from high-density strips to readout electronics is a non-trivial task. The ideal solution would be to integrate electronics with microstrip structure on the same Si wafer, although this will not be realized soon. Meanwhile, we take a different approach. Since ceramic substrates are widely used for packaging chips in the microelectronics industry, we are interested in building the MSGC on ceramics. Using multilayer ceramics, it is possible to connect high-density strips on one side to low-density multi-pin electronics chips on the other side. As a substrate, ceramics has additional advantages of good thermal conductivity, low thermal expansion, chemical resistence, and fracture resistence in comparison with glass.

We identified one type of ceramic substrate A445 (with 91\% Alumina) from Kyocera. It has a low bulk resistivity of $10^{12} \Omega \mathrm{cm}$ at $20^{\circ} \mathrm{C}$, but its surface is porous and rough, making it unsuitable for photolithography. A procedure of diamond polishing is still not able to smooth the surface because of its large grain size. We have, however, successfully used another ceramic substrate A201 (with 98\%

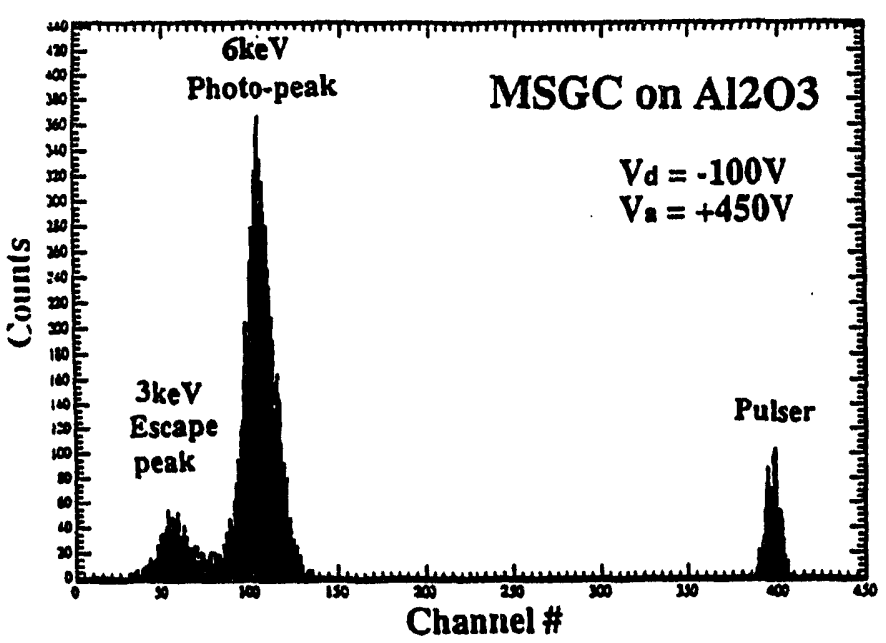

Figure 10: Pulse height spectra of ${ }^{55} \mathrm{Fe} X$-rays measured by the MSGC on A201 in P-10 gas. Bias voltages are indicated in the figure.

Alumina). Purer Alumina results in finer grain size that can be optically polished. Its surface is smooth enough for photolithography. To lower the resistivity, we used Boron ion implantation as suggested in Refs. $[2,5,7]$. By implanting $10^{16}$ ions $/ \mathrm{cm}^{2}$ at $150 \mathrm{keV}$ with an average range of 0.28 $\mu \mathrm{m}$ in Alumina, we measured the surface resistivity to be about $1.6 \times 10^{16} \Omega / \square$ (see Table 1).

We followed the same steps as in fabricating the MSGCs on glass. A layer of $2 \mu \mathrm{m} \mathrm{Al} \mathrm{thin} \mathrm{film} \mathrm{was} \mathrm{first} \mathrm{sput-}$ tered on the ceramic substrate. Due to the inferior surface quality of ceramics, the coated Al thin film was not as smooth as that on glass, which subsequently degraded the mask pattern transfer in photolithography to some extent. The Chlorine plasma etch was used to obtain the final microstrip plate. A microscope inspection showed that there were some defects such as indentation of strips and broken anode strips. Fig. 9 is a cross-section view of anode and cathode strips fabricated on the ceramic substrate. The surface seems to be not as smooth as that of glass (cf. Fig. 4). Nevertheless, the overall profile of anode and cathode strips is good. Most strips are actually uniform and of good quality.

Fig. 10 is the pulse height spectrum measured by the MSGC on alumina for ${ }^{35} \mathrm{Fe} \mathrm{X}$-rays together with an electronic pulser. The anode voltage is $+450 \mathrm{~V}$ and the drift cathode voltage is $-100 \mathrm{~V}$. Both the $6 \mathrm{keV}$ photo-peak and the $3 \mathrm{keV}$ escape peak are clearly resolved and well separated. The FWHM of the pulser peak is 10 channels, and the FWHM of the $6 \mathrm{keV}$ photo-peak is 18 channels. The intrinsic resolution (FWHM) at $6 \mathrm{keV}$ is $15.0 \%$. This energy resolution indicates that the strip uniformity on the ceramic substrate is not much worse than that on the glass substrate.

The gas gains of the MSGC on alumina were measured at anode voltages from $+400 \mathrm{~V}$ to $+530 \mathrm{~V}$. They are shown in Fig. 11 together with an exponential fit. Its gain dynamic 


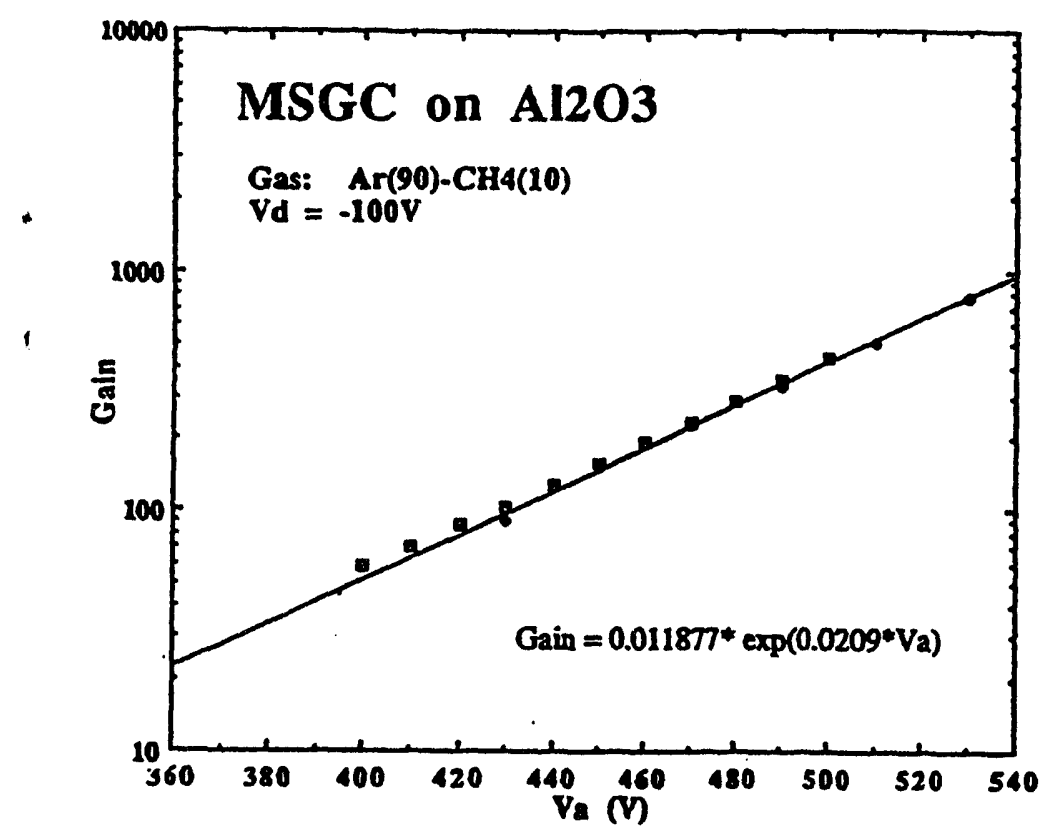

Figure 11: The gas gain dependence on anode voltages for the MSGC on A201 in P-10 gas. An Exponential fit is shown.

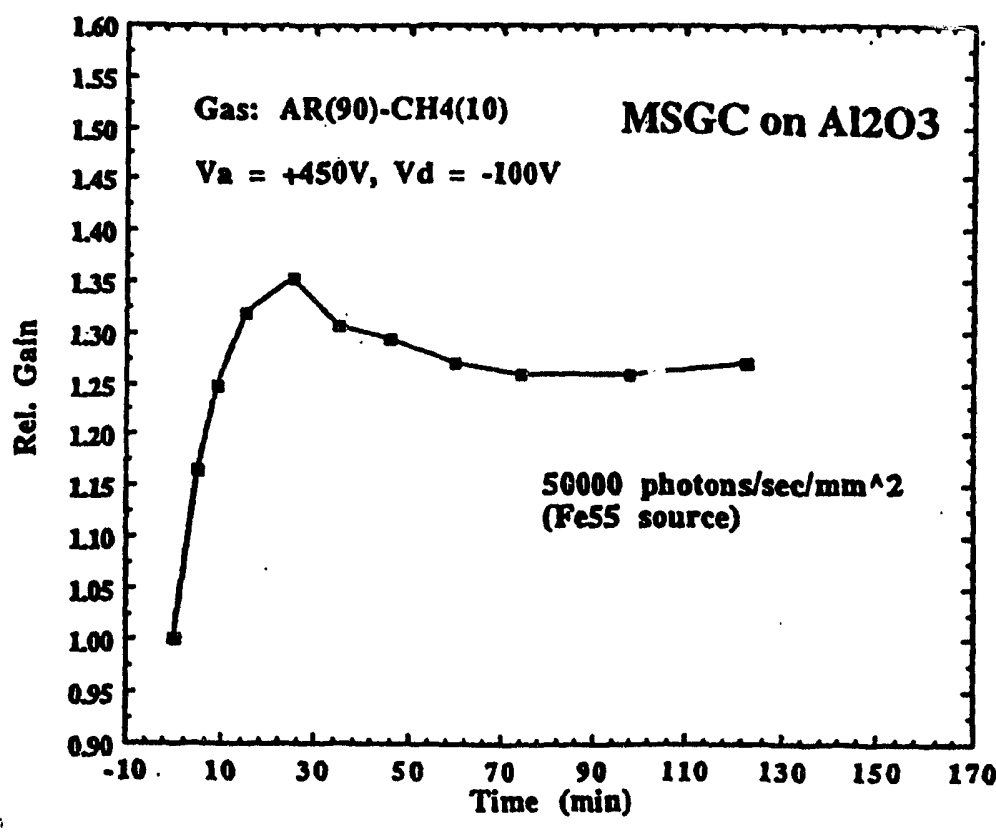

Figure 12: Time dependence of the relative gas gains for the MSGC on A201. It is monitored by a ${ }^{55} \mathrm{Fe}$ source with a flux of 50000 photons $/ \mathrm{sec} / \mathrm{mm}^{2}$. Bias voltages are indicated. range is very similar to those of MSGCs on glass as shown in Fig. 7. A maximum gain close to 1000 was reached before serious discharges occurred at $V_{a}=+540 \mathrm{~V}$.

Time dependence of the relative gas gain is shown in Fig. 12 for the MSGC on alumina (A201) at $V_{a}=+450 \mathrm{~V}$ and $V_{d}=-100 \mathrm{~V}$. It is obtained by monitoring the photopeak position for an ${ }^{55} \mathrm{Fe}$ source at a flux of 50000 photons $/ \mathrm{sec} / \mathrm{murn}^{2}$. We observed a large conditioning effect within the first hour immediately after biasing the anode strips. The pulse height increased significantly in the first 30 minutes and then decreased somewhat in the next 30 minutes. Afterwards, the gas gain stablized. This behavior may be related to surface charging after turning on the bias voltage, until an equilibrium condition is reached. Since the measured surface resistivity was still rather high $\left(1.6 \times 10^{16} \Omega / 0\right)$, a higher dosage of B-ion implantasion is needed to increase the surface conductivity so that the surface-charging effects can be reduced. Continued tests are underway to verify this point.

\section{CONClusion}

In summary, we have built high-quality microstrip gas chambers on glass and ceramic substrates using facilities at the Berkeley Microfabrication Laboratory. Typical energy resolutions of about 15\% have been achieved for 6 $\mathrm{keV} \mathrm{X}$-rays using P-10 gas at atmospheric pressure. Gas gains of 1000 can be obtained before serious discharges occurred. The maximum gains are limited by the quenching gas of $10 \% \mathrm{CH}_{4}$. Higher gains should be attainable with more quencher. Stable operation of the microstrip gas chamber can be maintained when the low resistive, electronic conduction substrate is choosen. Both Schott glass S8900 and Corning glass 7740 are sufficient for the moderate rate of 50000 photons $/ \mathrm{sec} / \mathrm{mm}^{2}$. Ceramic substrate is also promising for the microstrip gas chamber although its surface conductivity needs to be increased more with B-ion implantations.

\section{ACKNOWLEDGMENTS}

We acknowledge the use of the Berkeley Microfabrication Laboratory in this project. We thank Dr. Ronaldo Bellazzini and Dr. Gene Barasch for useful discussions and communications. We also thank Dr. James Symons and Dr. Art Poskanzer for their interest and support. Technical support by Fred Bieser and John Wolf are appreciated. W.G.G. thanks Dr. Eric Hjort for his help with Kmax.

This work was supported by the Director, Office of Energy Research, of the U.S. Department of Energy, under contract No. DE-AC03-76SF00098.

Reference to a company or product name does not imply approval or recommendation of the product by the U.S. Department of Energy to the exclusion of others that may be suitable. 


\section{REFERENCES}

[1] A. Oed. Position-Sensitive Detector with Microstrip Anode for Eletron Multiplication with Gases. Nucl. Instr. and Meth., A263:351-359, 1988.

[2] F. Angelini, et al. A Microstrip Gas Avalanche Chamber with Two-Dimensional Readout. Nucl. Instr. and Meth., A283:755-761, 1989.

[3] A. Oed, et al. A New Position Sensitive Proportional Counter with Microstrip Anode for Neutron Detection. Nucl. Instr. and Meth., A284:223-226, 1989.

[4] F. Hartjes, et al. A Prototype Microstrip Gas Detector. Nucl. Instr. and Meth., A289:384-387, 1990.

[5] F. Angelini, et al. The Microstrip Gas Chamber. Nucl. Phys. B, 23A:254-260, 1991.

[6] C. Budtz-Jorgensen, et al. Microstrip Proportional Counters for X-ray Astronomy. Nucl. Instr. and Meth., A310:82-87, 1991.

[7] F. Angelini, et al. Results from the First Use of Microstrip Gas Chambers in a High-Energy Physics Experiment. Nucl. Instr. and Meth., A315:21-32, 1992.

[8] C. Budtz-Jorgensen, Features of the Microstrip Proportional Counter Technology. Rev. Sci. Instrum. 63:648-658, 1992.

[0] R. Bouclier, et al. High Flux Operation of Microstrip Gas Chambers on Glass and Plastic Supports. Nucl. Instr. and Meth., A323:240-246, 1992.

[10] R. Bouclier, et al. Performance of Gas Microstrip Chambers on Glass Substrate with Electronic Conductivity. Nucl. Instr. and Meth., A332:100-106, 1993.

[11] G.D. Minakov, et al. Performance of Gas MicroStrip Chambers on Glass with Ionic and Electronic Conductivity. Nucl. Instr. and Meth., A326:566-569, 1993.

[12] F. Sauli, Collaboration Meeting of RD28: Development of Gas Micro-Strip Chamber for Radiation Detection and Tracking at High Rates. CERN, Geneva Switzerland, June 16, 1993.

[13] P. Geltonbort, editor, Workshop on Progress in Gaseous MicroStrip Proportional Chambers. Institut Laue - Langevin, Grenoble, France, June 21-23, 1993.

[14] R. Bouclier, et al. Development of Microstrip Gas Chambers on Substrate with Electronic Conductivity. IEEE 1993 Nuclear Science Symposium, San Francisco, Nov. 2-6, 1993.

[15] M. Salomon, et al. Some Properties of Gas Microstrip Detectors Made on Tedlar Substrate and Operating with $\mathrm{CF}_{4} /$ Isobutane Gas. IEEE 1993 Nuclear Science Symposium, San Francisco, Nov. 2-6, 1993. 

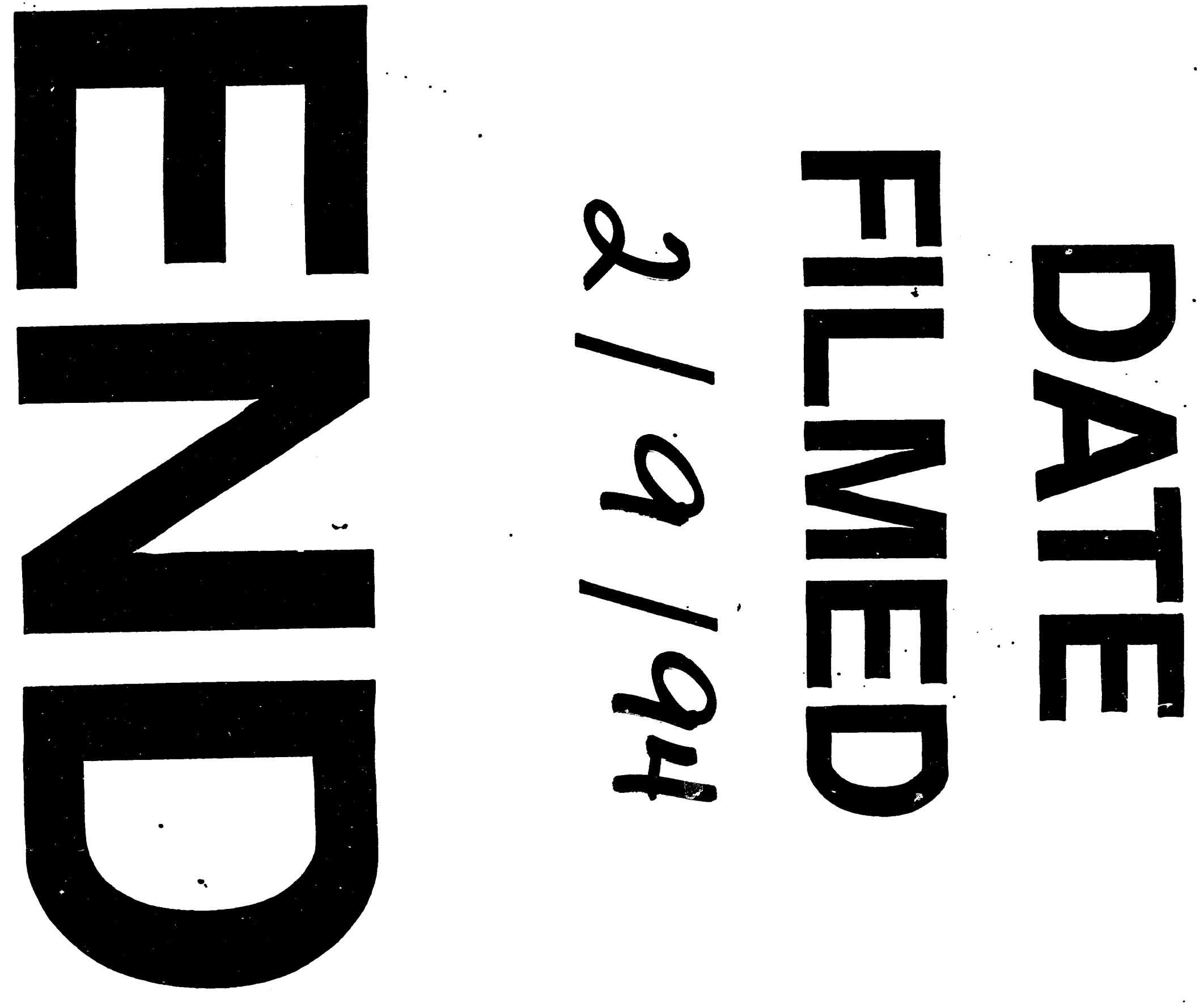


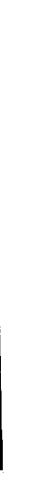

